\section{Reappraising insight in psychosis}

\author{
Multi-scale longitudinal study
}

MANUEL J. CUESTA, VICTOR PERALTA and AMALIA ZARZUELA

\section{Background Many patients suffering from psychosis are unaware of their disorder and symptoms.}

\begin{abstract}
Aims To investigate whether insight changes with time, and how it relates to patients' psychopathology, and to examine the correlations between insight scales in patients with psychoses.
\end{abstract}

Method Seventy-five consecutively admitted in-patients with schizophrenia, affective disorder with psychotic symptoms, or schizoaffective disorder were examined after remission of an acute episode and at follow-up ( $>6$ months). Three different scales were used to assess insight.

Results To some extent, insight into past episodes improved over time in patients with psychosis, regardless of diagnosis. Few significant relationships between insight and psychopathology remained stable at follow-up. The higher the negative and disorganisation dimensions at baseline, the less did attitudes to treatment vary when tested at follow-up. No predictive value for variability of psychopathological dimensions was found for insight dimensions. The insight scales used were highly intercorrelated, suggesting that they measure the same construct.

Conclusions Insight and

psychopathology seem to be semi-independent domains.

Declaration of interest Funded by the Spanish National Health Service (grant FIS 97/0480).
It is well-established that many patients with psychosis are unaware of their disorders and symptoms (World Health Organization, 1973). Moreover, insight is a clinical modulator of compliance with treatment and a good indicator of prognosis (McEvoy et al, 1989b; Buchanan, 1992; Kemp et al, 1996).

In the past decade, interest has increasingly focused on translating definitions of lack of insight into specific instruments, but until now, no consensus has been reached. However, multidimensional models have achieved agreement on the dimensions underlying it, and at least three (non-exclusive) are widely accepted: awareness of illness, awareness of symptoms, and cooperation with treatment.

We carried out a longitudinal study aimed at answering three questions. (a) Is the insight of patients with psychoses stable during the course of psychosis? (b) Are relationships between the psychopathological dimensions and the dimensions of lack of insight at baseline the same as during follow-up? (c) Are insight scales intercorrelated?

\section{METHOD}

\section{Sample}

Seventy-five consecutively admitted patients presenting at least one psychotic symptom were included in the study. They were evaluated at two different points: after remission of an acute episode $(n=75)$ and in a phase of clinical stabilisation after discharge (ranging from 6 months to 2 years) $(n=56) ; 19$ patients were not available for follow-up assessment. The subjects met criteria for DSM-IV (American Psychiatric Association, 1994) diagnoses of schizophrenia $(n=37)$, affective disorder with psychotic symptoms $(n=27) \quad$ or schizoaffective disorder $(n=11)$.

\section{Procedure and assessment measures}

Participants gave informed consent to enter the study. Patients with a history of an organic central nervous disorder, drug or alcohol misuse in the past year or learning disability were excluded. The Comprehensive Assessment Schedule History (CASH; Andreasen et al, 1992) was used as a semi-structured interview to assess psychopathology at baseline and at follow-up. Interrater reliability for positive and negative symptom scales has been reported as good to excellent (Peralta et al, 1992). Liddle's three-dimensional model (Liddle, 1987), with the addition of depressive and manic dimensions, was chosen for the analysis of psychopathological status.

Three instruments for insight assessment were used: the Scale to Assess Unawareness of Mental Disorder (SUMD) (Amador et al, 1991, 1994), the Insight and Treatment Attitudes Questionnaire (ITAQ) (McEvoy et al, 1981), and three insight items from the Manual for Assessment and Documentation in Psychopathology (AMDP) (Guy \& Ban, 1979). Insight assessment took place in two sessions; the AMDP insight items and the SUMD in the first session, and the ITAQ in the second. The SUMD was assessed by M.J.C., AMDP items were scored by M.J.C. and V.P. and the ITAQ by A.Z. For AMDP insight items, high interrater reliability between two raters has been reported previously (Cuesta \& Peralta, 1994). Each evaluator was blind to the results of the others.

The SUMD is a 20-item semi-structured interview which evaluates global insight, insight into illness and insight into symptoms. It comprises three ratings each for global insight into current and past illness: general awareness of having a mental disorder, need for psychiatric treatment, and social consequences of the disorder. Moreover, by averaging responses referring to 17 psychopathological signs and symptoms, which were scored on a 5-point scale, four additional scales were obtained: patients' current and past awareness, and current and past attributional patterns. In our study, 'past' ratings referred to the period of recent hospitalisation, and 'current awareness' was rated according to current state (i.e. moderate or severe) of positive and negative symptoms. The psychometric properties of the SUMD scale 
and comparison with other instruments have been described elsewhere (Amador et al, 1991; Amador \& Seckinger, 1997; Baier et al, 1998).

The ITAQ consists of a semi-structured interview of 11 items. Each item is scored from 0 (no insight) to 2 (good insight) and the total score is used as an insight measure. This questionnaire encompasses recognition of mental disorder (first five items) and attitudes to medication, hospitalisation and follow-up evaluation (six items) (McEvoy et al, 1989a).

The third instrument was extracted from the Spanish version of the Manual for Assessment and Documentation in Psychopathology (AMDP; López-Ibor, 1980). The AMDP is a comprehensive psychopathological inventory of 100 items. Symptoms are rated from 0 (absent) to 3 (severe). Although it is not a specific scale, it includes three items for the assessment of insight (not feeling ill, lack of insight and uncooperativeness), which partly resemble the three insight dimensions reported in David's model (David, 1990; David et al, 1992).

Patients were recruited for follow-up assessment after a period of at least 6 months; they had to have been clinically stabilised for at least 3 months. The same psychopathological and insight assessment procedures were administered at followup. If patients suffered another acute episode, then a further 6 months had to elapse before follow-up.

\section{Statistical analysis}

First, we inspected for any possible bias resulting from the fact that the follow-up sample was smaller, by comparing the baseline epidemiological, psychopathological and insight measures of patients who had not returned for follow-up with those who remained in the study. Parametric contrasts between baseline and follow-up insight scores were used to examine changes in insight over time in the total sample. To ascertain changes in insight longitudinally and any influences of patients' diagnosis, repeated measures of multivariate analysis of variance (MANOVA) were carried out, with diagnosis as the between-subject factor and time of assessment as the withinsubject factor. Longitudinal relationships between psychopathology and insight were analysed in three ways. First, crosssectional Pearson correlation coefficients between insight and psychopathological variables at baseline and follow-up were examined. Second, correlations between scores for changes in psychopathology and changes in insight were obtained. Third, multiple regression analysis assessed whether insight at baseline predicts a change in psychopathology and whether psychopathology at baseline predicts a change in insight at follow-up. A stepwise variable selection method was used; variables were entered into the model at a probability of $F=0.05$, and were removed at $F=0.1$.

\section{RESULTS}

The socio-demographic and psychopathological characteristics of the 19 patients who refused to participate in the follow-up assessment were very similar to those of the 56 patients who remained in the study, except for lower educational achievement in those who refused to participate $(t=2.44, P \leqslant 0.018)$. There were no differences in baseline insight measures between the two groups, except for the AMDP items of not feeling ill $(t=2.11$, $P \leqslant 0.03$ )

Psychopathological differences among the three groups of people with psychotic disorders were mostly due to the process of diagnostic classification. Schizoaffective patients were older $(F=3.38, P \leqslant 0.03)$ and had had a greater number of episodes $(F=8.11, P \leqslant 0.0007)$ than patients with schizophrenia or affective disorders (Table 1).

\section{Insight in the total sample and in diagnostic groups}

At baseline assessment, a large proportion of patients showed moderate to severe lack of awareness of having a mental disorder: the figure varied between $49 \%$ and $66 \%$, depending on the instrument used and the item considered.

Taking the sample as a whole, four SUMD measures showed a significant improvement over time, after applying the Bonferroni inequality correlation to account for a high number of statistical tests (Bland \& Altman, 1995). The $P$-value for statistical significance was $\leqslant 0.0035$, since 14 statistical procedures were run together. Patients improved in past awareness of mental disorder $(t=-3.57$, $P \leqslant 0.001)$, past awareness of the effect achieved by medication $(t=-3.68$, $P \leqslant 0.001)$, and past awareness of the social consequences of mental disorder $(t=-3.79, \quad P \leqslant 0.001)$. There was an improvement in current awareness of the social consequences of mental disorder $(t=-3.53, P \leqslant 0.001)$. The item 'not feeling ill' on the AMDP also showed improvement over time $(t=3.12, P \leqslant 0.003)$.

Significant differences between the diagnostic groups were obtained in three scores on the SUMD scale: current attribution $(F=7.66, P \leqslant 0.002)$, current awareness of mental disorder $(F=10.3, P \leqslant 0.001)$ and current awareness of the social consequences of mental disorder $(F=6.76$, $P \leqslant 0.003)$. In addition, there were significant differences in two AMDP items: not feeling ill $(F=6.69, P \leqslant 0.003)$ and lack of insight $(F=7.85, P \leqslant 0.001)$. Post-hoc contrasts demonstrated that patients with schizophrenia were more unaware than patients with affective disorders, though patients with schizophrenia were less aware than patients with schizoaffective disorder in only two insight ratings (current awareness of social consequences of mental disorder; and not feeling ill (AMDP), both at baseline assessment). No differences were found between schizoaffective and affective patients. Significant differences associated with improvement over time were only found in two SUMD items, namely past awareness of mental disorder $(F=11.9$, $P \leqslant 0.002$ ) and past awareness of the social consequences of mental disorder $(F=10.6, P \leqslant 0.002)$. There were no significant diagnosis-by-time interactions (Table 2).

\section{Dimensions of insight and psychopathological dimensions}

Cross-sectional Pearson correlation coefficients were examined between psychopathological and insight dimensions at both assessment points. The $P$-value for statistical significance after Bonferroni correction was set at $\leqslant 0.15$ (corresponding to $P \leqslant 0.002$ for $5 \times 14$ correlation matrices), since a more liberal statistical criterion is allowed in exploratory analysis (Bland \& Altman, 1995). At baseline assessment the psychotic dimension was significantly associated with greater disturbances in both current $(r=0.47,0.27$ $0.63, P \leqslant 0.001)$ and past attribution of illness $(r=0.43,0.22-0.59, P \leqslant 0.001)$. The negative dimension was related to greater disturbances in current awareness of mental disorder $(r=-0.41,0.20-0.58, P \leqslant 0.001)$, current awareness of effect achieved by 
Table I Socio-demographic and clinical characteristics of the sample

\begin{tabular}{|c|c|c|c|c|c|c|}
\hline & Total group $n=75(100 \%)$ & $S Z n=37(49.3 \%)$ & $A D n=27(36.0 \%)$ & SAD $n=I I(14.7 \%)$ & \multicolumn{2}{|c|}{ Difference between groups } \\
\hline & \multirow[t]{2}{*}{ Mean (s.d.) } & \multirow[t]{2}{*}{ Mean (s.d.) } & \multirow[t]{2}{*}{ Mean (s.d.) } & \multirow[t]{2}{*}{ Mean (s.d.) } & & \\
\hline & & & & & $F / P$ & Contrast \\
\hline Age & $34.04(9.60)$ & $32.24(9.10)$ & $33.85(9.60)$ & $40.55(9.25)$ & $3.38 / 0.03$ & $S A D>S Z$ \\
\hline Education (years) & $10.35(3.03)$ & $10.81(3.49)$ & $9.67(2.18)$ & $10.45(3.14)$ & $1.19 / 0.30$ & - \\
\hline No. of episodes & $5.36(5.36)$ & 4.14 (3.49) & 4.78 (4.77) & $10.82(8.32)$ & $8.11 / 0.0007$ & $S A D>S Z, A D$ \\
\hline \multirow[t]{2}{*}{ Age at onset } & $24.59(7.21)$ & $23.46(6.50)$ & $26.26(8.05)$ & $24.27(7.21)$ & I.12/0.33 & None \\
\hline & & & & & $\chi^{2} / P$ & Contrast \\
\hline Male (\%) & 47 (62.7\%) & $24(64.9 \%)$ & $15(55.6 \%)$ & $8(72.7 \%)$ & $1.12 / 0.57$ & None \\
\hline Single (\%) & $54(72.0 \%)$ & 30 (81.I\%) & $16(59.3 \%)$ & $8(72.7 \%)$ & $3.15 / 0.20$ & None \\
\hline \multicolumn{4}{|c|}{ Baseline assessment of psychopathological dimensions } & & $F / P$ & Contrast \\
\hline Psychotic & $1.10(1.22)$ & $1.56(1.25)$ & $0.56(0.85)$ & $0.86(1.38)$ & $5.97 / 0.004$ & $S Z>A D$ \\
\hline Negative & $1.53(1.13)$ & $2.03(0.99)$ & $0.74(0.97)$ & $1.73(0.93)$ & $|3.64 / 0.000|$ & $S Z, S A D>A D$ \\
\hline Disorganisation & $0.72(0.86)$ & $0.96(1.02)$ & $0.38(0.47)$ & $0.67(0.82)$ & $3.70 / 0.02$ & $S Z>A D$ \\
\hline Depression & $0.53(0.76)$ & $0.24(0.49)$ & $0.59(0.75)$ & $1.36(0.92)$ & $12.18 / 0.000 \mid$ & $S A D>S Z, A D$ \\
\hline Mania & $0.24(0.49)$ & $0.05(0.23)$ & $0.48(0.58)$ & $0.27(0.65)$ & $6.96 / 0.001$ & $A D>S Z$ \\
\hline \multicolumn{4}{|c|}{ Follow-up assessment of psychopathological dimensions } & & $F / P$ & Contrast \\
\hline Psychotic & $0.79(1.14)$ & $1.22(1.35)$ & $0.16(0.37)$ & $0.69(0.80)$ & $6.02 / 0.004$ & $S Z>A D$ \\
\hline Negative & $1.36(1.10)$ & $1.97(1.02)$ & $0.4 I(0.6 I)$ & $1.44(0.69)$ & $18.91 / 0.000 \mid$ & $S Z, S A D>A D$ \\
\hline Disorganisation & $0.39(0.68)$ & $0.68(0.84)$ & $0.09(0.22)$ & $0.08(0.15)$ & $6.20 / 0.003$ & $S Z>A D$ \\
\hline Depression & $0.48(0.79)$ & $0.48(0.83)$ & $0.47(0.84)$ & $0.50(0.53)$ & $0.003 / 0.99$ & None \\
\hline Mania & $0.05(0.30)$ & $0.03(0.19)$ & $0.11(0.46)$ & $0.00(0.00)$ & $0.49 / 0.62$ & None \\
\hline
\end{tabular}

SZ, schizophrenia; AD, affective disorder; SAD, schizoaffective disorder.

medication $(r=0.41,0.20-0.58, P \leqslant 0.001)$, current awareness of the social consequences of mental disorder $(r=0.44$, $0.23-0.60, P \leqslant 0.001$ ), and with two items of the AMDP: not feeling ill $(r=0.35$, $0.13-0.53, P \leqslant 0.002)$ and lack of insight $(r=0.39,0.17-0.56, P \leqslant 0.002)$. The only item to which the disorganisation dimension was significantly related was disturbances on current awareness of effect achieved by medication $(r=-0.39,0.17-$ $0.56, P \leqslant 0.001)$. No significant associations were found between insight scores and affective dimensions. At follow-up the pattern of associations was rather different, since only negative and disorganisation dimensions were significantly associated with failure of insight. The negative dimension was significantly associated with lack of current awareness of mental disorder $(r=0.41,0.16-0.60, P \leqslant 0.002)$ and not feeling ill $(r=0.40,0.15-0.60, P \leqslant 0.002)$; and the disorganisation dimension with lack of awareness of past mental disorders ( $r=0.52,0.29-0.68, P \leqslant 0.001$ ), awareness of effect achieved by medication (current $r=0.40,0.15-0.60, P \leqslant 0.0002$ and past $r=0.47,0.23-0.65, P \leqslant 0.002)$ and current awareness of the social consequences of mental disorder $(r=0.48, \quad 0.24-0.66$, $P \leqslant 0.001$ ) (Table 3).

To find out whether changes in insight might be accounted for by improvement in clinical status, correlations between change scores in psychopathological dimensions (baseline minus follow-up psychopathological dimensions) and change scores in insight measures (baseline minus follow-up insight scores) were obtained. All correlation coefficients were lower than $r=0.48(P \leqslant 0.005)$, when the critical value for $P \leqslant 0.005$ after Bonferroni correction was $\leqslant 0.0035$.

To assess whether variability in psychopathology dimensions and in insight disturbances differed from each other, multiple regression analysis (stepwise method) was used. Specifically, we tested whether insight at baseline predicts any change in psychopathology dimensions over time, and whether psychopathology at baseline predicts change in insight over time. To avoid the use of a large number of statistical procedures regarding insight, the 14 insight measures were condensed through factor analysis, by the principal components method with an oblimin rotation. Two factors were obtained at baseline $(76.9 \%$ explained variance), representing a general awareness dimension, with high loading on all SUMD items (eigenvalue $=9.44,67.5 \%$ explained variance), and a second factor reflecting attitudes to treatment, which had high weightings on the ITAQ total score and the AMDP refusal of treatment item, and moderate loading of the AMDP not feeling ill and the AMDP lack of insight items (eigenvalue $=1.31,9.4 \%$ explained variance). Both factors showed a considerable overlap, since they were highly associated $(r=-0.42)$. Factor scores of insight at baseline were included as dependent variables in separate equation regressions and each variance score of the five psychopathological dimensions (baseline minus follow-up scores), as independent variables. Neither of the two insight 


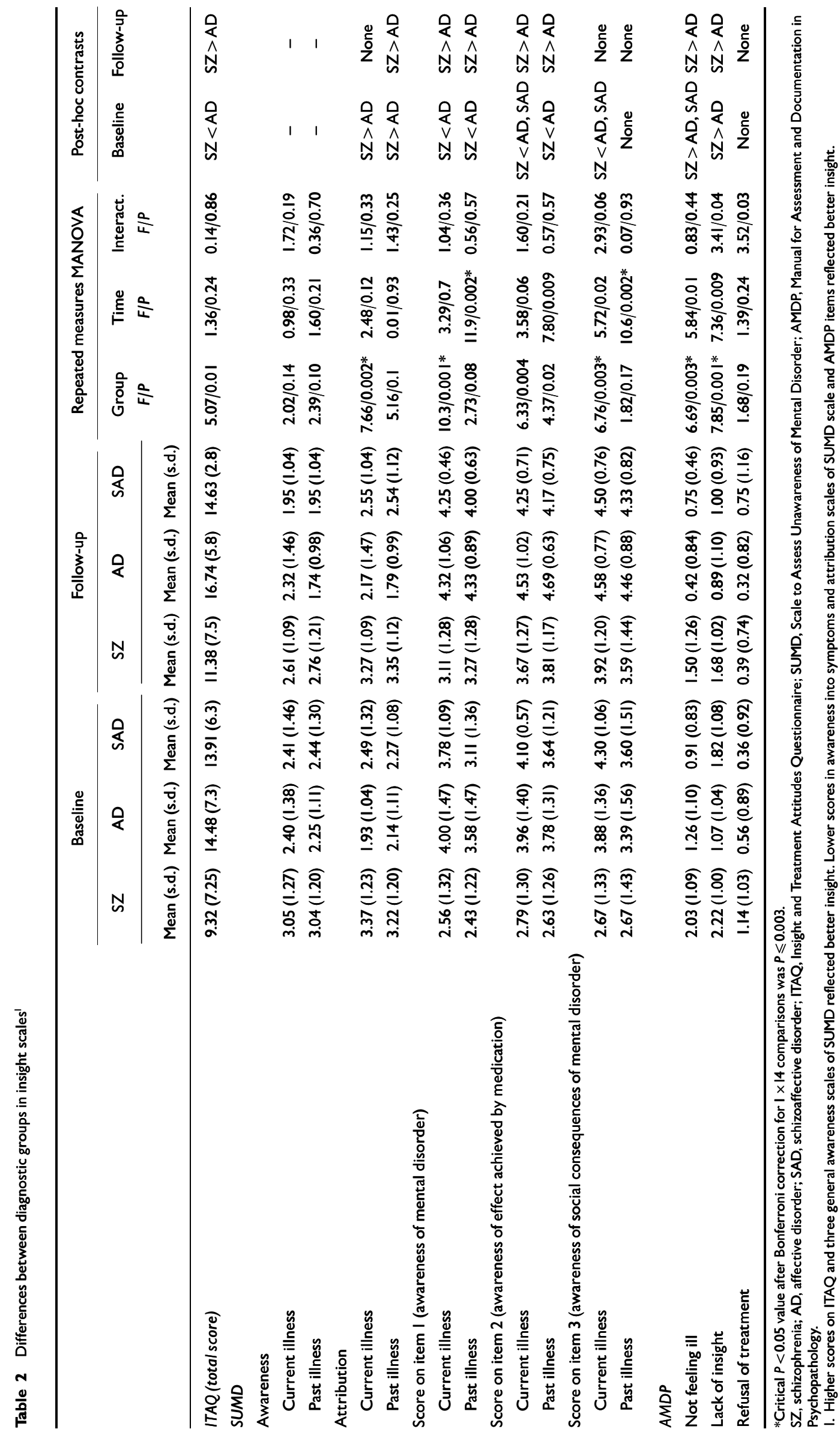


Table 3 Pearson correlation coefficients between insight and psychopathological dimensions at baseline $\left(r_{0}\right)$ and at follow-up assessment $\left(r_{1}\right)$

\begin{tabular}{|c|c|c|c|c|c|}
\hline & \multicolumn{5}{|c|}{ Psychopathological dimension $\left(r_{0} / r_{1}\right)$} \\
\hline & Psychotic & Negative & Disorganisation & Depression & Mania \\
\hline \multicolumn{6}{|l|}{ ITAQ } \\
\hline I. Total score & $-0.16 /-0.19$ & $-0.16 /-0.35$ & $-0.20 /-0.27$ & $0.04 / 0.15$ & $0.17 /-0.15$ \\
\hline \multicolumn{6}{|l|}{ SUMD } \\
\hline \multicolumn{6}{|l|}{ 2. Awareness total score } \\
\hline Current illness & $0.33 / 0.08$ & $0.31 / 0.25$ & $0.33 / 0.37$ & $-0.09 /-0.22$ & $-0.21 / 0.07$ \\
\hline Past illness & $0.27 / 0.40$ & $0.26 / 0.34$ & $0.30 / 0.41$ & $-0.07 /-0.34$ & $-0.13 / 0.12$ \\
\hline \multicolumn{6}{|l|}{ 3. Attribution total score } \\
\hline Current illness & $0.47 * / 0.08$ & $0.35 / 0.11$ & $0.12 / 0.12$ & $-0.06 /-0.26$ & $-0.26 / 0.14$ \\
\hline Past illness & $0.43^{*} / 0.34$ & $0.35 / 0.40$ & $0.13 / 0.35$ & $-0.004 /-0.27$ & $-0.21 / 0.15$ \\
\hline \multicolumn{6}{|c|}{ 4. Awareness of mental disorder } \\
\hline Current illness & $-0.29 /-0.26$ & $-0.4 I * \mid-0.4 I *$ & $-0.29 /-0.32$ & $0.15 / 0.17$ & $0.23 /-0.15$ \\
\hline Past illness & $-0.14 /-0.31$ & $-0.19 /-0.30$ & $-0.17 /-0.52 *$ & $0.007 / 0.40$ & $0.23 /-0.09$ \\
\hline \multicolumn{6}{|c|}{ 5. Awareness of effect achieved by medication } \\
\hline Current illness & $-0.29 /-0.13$ & $-0.4 I^{*} \mid-0.33$ & $-0.39 * /-0.40 *$ & $0.12 / 0.05$ & $0.29 /-0.22$ \\
\hline Past illness & $-0.23 /-0.31$ & $-0.28 /-0.30$ & $-0.23 /-0.47 *$ & $0.07 / 0.40$ & $0.23 /-0.33$ \\
\hline \multicolumn{6}{|c|}{ 6. Awareness of social consequences of mental disorder } \\
\hline Current illness & $-0.31 /-0.04$ & $-0.44 * /-0.30$ & $-0.33 /-0.48 *$ & $0.06 / 0.12$ & $0.27 /-0.10$ \\
\hline Current illness & $-0.19 /-0.11$ & $-0.24 /-0.20$ & $-0.2 \mathrm{I} /-0.4 \mathrm{I}$ & $0.01 / 0.35$ & $0.12 /-0.12$ \\
\hline \multicolumn{6}{|l|}{$A M D P$} \\
\hline 7. Not feeling ill & $0.26 / 0.27$ & $0.35 * / 0.40 *$ & $0.19 / 0.29$ & $-0.21 /-0.27$ & $-0.20 / 0.21$ \\
\hline 8. Lack of insight & $0.33 / 0.28$ & $0.39 * / 0.33$ & $0.15 / 0.22$ & $0.002 /-0.13$ & $-0.25 / 0.11$ \\
\hline 9. Refusal of treatment & $0.02 / 0.08$ & $0.29 / 0.01$ & $0.10 / 0.01$ & $-0.18 /-0.03$ & $-0.12 /-0.01$ \\
\hline
\end{tabular}

*Critical $P \leqslant 0.15$ value after Bonferroni correction for $5 \times 14$ matrix was $P \leqslant 0.002$.

$95 \% \mathrm{Cl}$ for significant associations at baseline assessment $(n=75)$ ranged from $r=0.35(0.13-0.53)$ to $r=0.47(0.27-0.63)$.

$95 \% \mathrm{Cl}$ for significant associations at follow-up assessment $(n=56)$ ranged from $r=0.40(0.15-0.60)$ to $r=0.52(0.29-0.68)$.

ITAQ, Insight and Treatment Attitudes Questionnaire; SUMD, Scale to Assess Unawareness of Mental Disorder; AMDP, Manual for Assessment and Documentation in

Psychopathology.

summary scores predicted changes in psychopathological dimensions. A second set of multiple regression analyses was conducted to test whether psychopathological dimensions at baseline predict change in insight over time. Higher levels of negative dimension at baseline $\left(B=0.33,0.15-0.50, R^{2}=0.21\right.$, $F=14.67, P=0.0003)$ and higher levels of disorganisation dimension at baseline $\left(B=0.21, \quad 0.06-0.35, \quad R^{2}=0.14, \quad F=8.62\right.$, $P=0.0049$ ) predicted lower degrees of variance in the attitudes to treatment dimension of insight.

\section{Relationships between insight scales}

To verify whether baseline insight scores would predict identical insight scores at follow-up, multiple regressions were performed. Baseline measures of insight were good predictors of identical followup insight score, ranging between $R^{2}=0.26$ and $R^{2}=0.44 . R^{2}$ coefficients were statistically significant after Bonferroni correction, with the exception of the AMDP 'refusal of treatment' item, which did not enter the regression equation $\left(R^{2}=0.25\right.$, $P \leqslant 0.059$ ). Correlational analysis between identical measures of insight at the two assessment points gave similar results (data shown in bold in the diagonal of Table 4). Moreover, scales of insight were highly intercorrelated, both at baseline and at follow-up. Correlation coefficients ranged from $r=0.35$ and $r=0.86$ at baseline assessment, and between $r=0.55$ and $r=0.89$ at follow-up, except for the refusal of treatment item, which showed few significant associations with the remaining insight measures (Table 4).

\section{DISCUSSION}

\section{Insight in total sample and in diagnostic groups}

This study demonstrated that moderate to severe disturbances of insight persisted over time, and that the level of insight into their present illness was not significantly improved in patients suffering from functional psychoses. Depending on the insight scale used, between $29 \%$ and $49 \%$ of these patients continued to have only fair to poor insight at the followup assessment.

As regards diagnosis, patients suffering from schizophrenia showed poorer insight than patients with affective disorders but few significant differences from schizoaffective sufferers. Our results not only reemphasised that lack of insight is a prevalent feature of functional psychoses, as 
Table 4 Pearson correlation coefficients between instruments of insight at baseline assessment ( $r_{0}$, inferior triangle) and between insight instruments at follow-up assessments $\left(r_{\mathrm{l}}\right.$, superior triangle)

\begin{tabular}{|c|c|c|c|c|c|c|c|c|c|c|c|c|c|c|c|}
\hline \multirow{3}{*}{$\begin{array}{l}\text { Baseline } \\
\text { assessments }\end{array}$} & & \multicolumn{14}{|c|}{ Follow-up assessments } \\
\hline & & \multirow{2}{*}{$\begin{array}{c}\text { ITAQ } \\
\text { I }\end{array}$} & \multicolumn{10}{|c|}{ SUMD } & \multicolumn{3}{|c|}{ AMDP } \\
\hline & & & 2 & 3 & 4 & 5 & 6 & 7 & 8 & 9 & 10 & II & 12 & 13 & 14 \\
\hline \multirow[t]{4}{*}{ ITAQ } & I & $0.62 *$ & -0.51 & -0.54 & $-0.63^{*}$ & $-0.60 *$ & $0.66 *$ & 0.44 & $0.64 *$ & $0.54 *$ & $0.61 *$ & $0.58 *$ & $-0.68 *$ & $-0.65^{*}$ & -0.25 \\
\hline & 3 & $-0.46^{*}$ & $0.86 *$ & $0.51 *$ & 0.55 & $0.86 *$ & $-0.79 *$ & $-0.89 *$ & -0.48 & $-0.70 *$ & -0.55 & $0.65^{*}$ & $0.74 *$ & $0.79 *$ & 0.10 \\
\hline & 4 & $-0.56 *$ & $0.76^{*}$ & $0.68^{*}$ & $0.67 *$ & $0.72 *$ & $-0.69 *$ & -0.60 & $-0.56 *$ & -0.54 & $-0.74 *$ & $-0.7 I^{*}$ & $-0.68 *$ & $0.70 *$ & 0.03 \\
\hline & 5 & $-0.62^{*}$ & $0.60 *$ & $0.63 *$ & $0.86 *$ & $0.64^{*}$ & $-0.77 *$ & $-0.74 *$ & $-0.57^{*}$ & $-0.69 *$ & $-0.60 *$ & $-0.63 *$ & 0.73* & $0.78 *$ & 0.12 \\
\hline \multirow[t]{5}{*}{ SUMD } & 6 & $0.57^{*}$ & $-0.64^{*}$ & $-0.58^{*}$ & $-0.79 *$ & $-0.66 *$ & $0.64^{*}$ & $0.86^{*}$ & $0.71 *$ & $0.70 *$ & $0.7 I *$ & $0.64^{*}$ & $-0.8 I^{*}$ & $-0.85^{*}$ & -0.34 \\
\hline & 7 & $0.59 *$ & $-0.58 *$ & $-0.66^{*}$ & $-0.67^{*}$ & $-0.7 \mathrm{I} *$ & $0.74 *$ & $0.63^{*}$ & $0.55^{*}$ & $0.75^{*}$ & $0.64^{*}$ & $0.7 I^{*}$ & $-0.75^{*}$ & $-0.74^{*}$ & -0.17 \\
\hline & 8 & $0.46 *$ & $-0.66^{*}$ & $-0.56^{*}$ & $-0.74 *$ & $-0.62 *$ & $0.85^{*}$ & $0.64 *$ & $0.60 *$ & $0.83 *$ & $0.76 *$ & $0.7 I^{*}$ & $-0.63^{*}$ & $-0.63^{*}$ & $-0.60 *$ \\
\hline & 10 & $0.52 *$ & $-0.63^{*}$ & $-0.61 *$ & $-0.66^{*}$ & $-0.64^{*}$ & $0.81 *$ & $0.64^{*}$ & $0.81 *$ & $0.65^{*}$ & $0.53^{*}$ & $0.84 *$ & $-0.72^{*}$ & $-0.59 *$ & -0.23 \\
\hline & II & $0.58^{*}$ & $-0.62^{*}$ & $-0.72^{*}$ & $-0.6 I^{*}$ & $-0.73 *$ & $0.69 *$ & $0.83^{*}$ & $0.67^{*}$ & $0.77^{*}$ & $0.82 *$ & $0.66 *$ & $-0.64^{*}$ & $-0.59 *$ & -0.17 \\
\hline \multirow[t]{3}{*}{ AMDP } & 12 & $-0.48 *$ & 0.40 & $0.42 *$ & $0.54^{*}$ & $0.49^{*}$ & $-0.59 *$ & $-0.45^{*}$ & $-0.59 *$ & $-0.52^{*}$ & $-0.6 I^{*}$ & $-0.5 I^{*}$ & $0.62 *$ & $0.78^{*}$ & 0.22 \\
\hline & 13 & $-0.63^{*}$ & $0.59 *$ & $0.50 *$ & $0.73^{*}$ & $0.60 *$ & $-0.64^{*}$ & $-0.55^{*}$ & $-0.60 *$ & $-0.52 *$ & $-0.53^{*}$ & $-0.5 I^{*}$ & $0.66 *$ & $0.60 *$ & 0.34 \\
\hline & 14 & $-0.42^{*}$ & 0.28 & 0.19 & 0.35 & 0.31 & $-0.43^{*}$ & -0.35 & $-0.45^{*}$ & -0.39 & $-0.4 I^{*}$ & $-0.43^{*}$ & $0.43^{*}$ & $0.56^{*}$ & 0.25 \\
\hline
\end{tabular}

ITAQ, Insight and Treatment Attitudes Questionnaire; SUMD, Scale to Assess Unawareness of Mental Disorder; AMDP, Manual for Assessment and Documentation in Psychopathology.

I. ITAQ; 2. Awareness, current illness; 3. Awareness, past illness; 4. Attribution, current illness; 5 . Attribution, past illness; 6 . Awareness of mental disorder, current illness;

7. Awareness of mental disorder, past illness; 8 . Awareness of effect achieved by medication, current illness; 9 . Awareness of effect achieved by medication, past illness;

10. Awareness of social consequences of mental disorder, current illness; II. Awareness of social consequences of mental disorder, past illness; 12. Not feeling ill; 13. Lack of insight;

14. Refusal of treatment.

*Critical $P$ value after Bonferroni correction $P \leqslant 0.05(14 \times 14) / 2, P \leqslant 0.0005$

Diagonal in bold displaying inter-correlations between identical measures of insight at the two time points.

$95 \% \mathrm{Cl}$ for significant associations at baseline assessment $(n=75)$ ranged from $r=0.42(0.21-0.59)$ to $r=0.86(0.78-0.90)$.

$95 \% \mathrm{Cl}$ for significant associations at follow-up assessment $(n=56)$ ranged from $r=0.54(0.32-0.70)$ to $r=89(0.81-0.93)$.

cross-sectional studies have found (McEvoy et al, 1989a,b; Amador \& Strauss, 1993; Cuesta \& Peralta, 1994; Amador et al, 1994; David et al, 1995; Jorgensen, 1995; Kemp \& Lambert, 1995; Sanz et al, 1998), but also provided evidence of improvement over time in certain dimensions of insight.

There is, at first glance, a contradiction between an improvement in global insight measures in the total sample and the conclusions drawn from the analysis of diagnostic groups, since whenever the three different diagnostic groups were considered, most improvements vanished. Nevertheless, certain aspects of awareness regarding the past episode (unawareness of the effect achieved by medication and unawareness of the consequences of mental disorder) significantly improved in the long term when compared with the baseline assessment. It seems that patients with psychotic disorders were more concerned with particular circumstances of the past episode, such as the need for treatment and the need for admission, than with complete insight into their illness or symptoms. This agrees with the findings of a study which demonstrated an improvement over time on SUMD misattribution of past symptoms only in those patients who had been hospitalised (Smith et al, 1998).

\section{Dimensions of insight and psychopathological dimensions}

Psychopathological dimensions and insight did not show an identical pattern of relationships at the two assessment points, and no cross-sectional associations between changes in scores of insight and changes in scores of psychopathological dimensions were found. Only three out of 14 insight ratings showed stable significant relationships with psychopathological dimensions: 'current awareness of mental disorder' and the AMDP 'not feeling ill' item with the negative dimension, and 'current awareness of the effect achieved by medication' with the disorganisation dimension. Otherwise, it was found that baseline negative and disorganisation dimensions seemed to account for the variability in the attitudes to the treatment dimension at follow-up. However, psychopathological dimensions seemed to be independent of a general awareness insight dimension, and baseline insight factor scores were not predictive of any change in psychopathological dimensions. The data suggest that insight dimensions are correlated to a certain extent with negative and disorganisation dimensions, and that the remitting course of insight dimensions differed from that of positive and affective symptoms. Moreover, there were semiindependent longitudinal relationships between negative and disorganisation dimensions and one of the two insight dimensions, since these psychopathological dimensions showed unidirectional predictive associations with 'attitudes to treatment'. These semi-independent patterns of associations between the insight and 
psychopathological dimensions partly confirm our previous hypothesis that psychopathological and insight dimensions are unrelated domains (Cuesta \& Peralta, 1994; David et al, 1995). Moreover, studies reporting factor analysis of the whole range of psychotic symptoms, where the insight dimension was extracted as an independent dimension with respect to psychotic and affective symptoms (Peralta \& Cuesta, 1994; van Os et al, 1996) provided support for at least partial independence of insight and psychopathology. In addition, pooling together this study's baseline assessments of insight and psychopathology (14 insight baseline scorings and five psychopathological dimensions) and performing a factor analysis by the principal components methods, we extracted four factors $(76.2 \%$ of explained variance) (data not shown): (by order of appearance) general awareness, positive-negative-disorganisation, refusal of treatment (loading significantly only this item), and a depressive-manic factor. However, other factors contributing to insight, such as preexisting attitudes, cannot be disregarded in this study, since it has been reported that personality traits may to some extent explain the variability of insight in schizophrenia (Lysaker et al, 1999).

An alternative explanation for the scarcity of stable associations may be that the relationships between insight and symptoms are not always linear. To examine this possibility, associations between ITAQ total score and psychopathological domains were inspected using curve-fit analysis procedures, and the curvilinear fits obtained were non-significant.

Unlike other studies (Kim et al, 1997), in this study we detected no associations between affective dimensions and insight. However, other authors have reported that depressed mood enables patients to understand their disorder better (Amador et al, 1991; Sanz et al, 1998; Smith et al, 1998). Two explanations might account for this discrepancy. First, it has been reported that insight instruments are not all equally able to detect associations with depressed mood (Sanz et al, 1998). Second, relationships between depressive mood and increasing awareness of symptoms and disorder may be somewhat tautological, since patients who are more aware of their disturbances are also more likely to be depressed (Amador et al, 1994).

\section{Relationships between insight scales}

Results of insight measures at baseline were highly correlated with those of the same insight measures at follow-up, except for baseline refusal of treatment (AMDP). However, only weak significant correlations have been reported in another longitudinal study (David et al, 1995). Differences in design between the two studies may account for this discrepancy, since David and colleagues assessed insight during an acute episode, and in our study this assessment was performed after remission of the acute episode. Moreover, insight scales achieved concurrent validity, since, in the absence of a 'gold standard' for measuring insight, scores from different scales with an equivalent purpose and content were strongly associated (Salvador-Carulla, 1996).

Therefore, as far as the insight scales are concerned, it seems that the discrepancies found between studies are due more to methodological factors, such as selection of patients or course and phase of illness, than to great differences between the scales. This suggests that the choice of a particular insight scale is a question of the aims and preferences of investigators or clinicians. Nevertheless, the information gathered through multi-dimensional instruments better reflects the different components of the construct 'insight', and their use should be encouraged. Those instruments, such as the SUMD and SAI scales (Schedule of Assessment of Insight: David, 1990), permit a deeper understanding of relationships with other psychopathological domains, and, in the case of the SUMD scale, enable us to detect improvement in insight into past episodes. On the other hand, the ITAQ, which includes many items referring to treatment compliance, may be more appropriate for surveillance of some patients. Finally, multi-dimensional instruments provide a privileged approach for research on associations between insight dimensions and putative neurobiological processes.

\section{Limitations}

Certain limitations of the present study must be borne in mind. First, the insight scales were administered blind with respect to each other, except for the SUMD scale, which was evaluated by only one rater, who was not completely blind to the assessment of insight with the AMDP inventory.
However, when the sample was split into those patients assessed by the partially blind rater and those evaluated by the completely blind rater on the SUMD scale, the results did not change substantially.

Second, the evaluators of psychopathology were not blind to insight assessments; however, it is impossible to assess insight without knowing the psychopathological status. This potential bias could only be solved by means of selfassessment instruments, but all that is available is a brief and limited self-report scale for the assessment of insight (Birchwood et al, 1994).

Third, the period between the two evaluations varied substantially across the sample (between 6 months and 2 years). However, in our study we emphasised the stability of psychotic symptoms, in order to carry out the assessment sessions outside acute episodes.

Fourth, one quarter of the patients in the sample were not available for the second assessment. This is quite usual in follow-up studies of patients with psychosis, but limits the generalisability of our results, since one would suppose that more severely ill patients were more likely to have been lost to follow-up. In this respect, we could not find significant differences in psychopathological backgrounds between those patients who continued, and those who discontinued, the study, except for a slight but significant increase in one insight item (not feeling ill).

Finally, two statistical points should be borne in mind. Certain results from correlational procedures should be considered as exploratory, because of the large number of correlation analyses carried out, and definitive confirmation of insight as independent of symptoms awaits studies using confirmatory factor analysis.

\section{REFERENCES}

Amador, X. F., Strauss, D. H., Yale, S. A., et al (1991) Awareness of illness in schizophrenia. Schizophrenic Bulletin, 17, 113-132.

_ \& _ (1993) Poor insight in schizophrenia. Psychiatric Quarterly, 64, 305-318.

_, Flaum, M., Andreasen, N. C., et al (1994)

Awareness of illness in schizophrenia and schizoaffective and mood disorders. Archives of General Psychiatry, 5I, 826-836.

— \& Seckinger, R. A. (1997) The assessment of insight: a methodological review. Psychiatric Annals, 27, 798-805 
American Psychiatric Association (1994) Diagnostic and Statistical Manual of Mental Disorders (4th edn) (DSM-IV). Washington, DC: APA

Andreasen, N. C., Flaum, M. \& Arndt, S. (1992) The Comprehensive Assessment of Symptoms and History (CASH): an instrument for assessing diagnosis and psychopathology. Archives of General Psychiatry, 49 615-623.

Baier, M., Murray, R. L. E. \& McSweeney, M. (1998) Conceptualization and measurement of insight. Archives of Psychiatric Nursing, 12, 32-40.

Birchwood, M., Smith, J., Drury, V., et al (1994) A self-report Insight Scale for psychosis: reliability, validity and sensitivity to change. Acta Psychiatrica Scandinavica, 89, 62-67.

Bland, J. M. \& Altman, D. G. (1995) Multiple significance tests: the Bonferroni method. British Medical Journal, 310, 170.

Buchanan, A. (1992) A two-year prospective study of treatment compliance in patients with schizophrenia. Psychological Medicine, 22, 787-797.

Cuesta, M. J. \& Peralta, V. (1994) Lack of insight in schizophrenia. Schizophrenia Bulletin, 20, 359-366.

David, A. S. (1990) Insight and psychosis. British Journa of Psychiatry, 156, 798-808.

_, Buchanan, A., Reed, A., et al (1992) The assessment of insight in psychosis. British Journal of Psychiatry, 161, 599-602.

_, van Os, J., Jones, P., et al (1995) Insight and psychotic illness. Cross-sectional and longitudinal associations. British Journal of Psychiatry, 167, 621-628.

Guy, W. \& Ban, T. A. (1979) The AMDP System. Berlin: Springer

Jorgensen, P. (1995) Recovery and insight in schizophrenia. Acta Psychiatrica Scandinavica, 92 436-440.

Kemp, R. \& Lambert, T. J. C. (1995) Insight in schizophrenia and its relationship to psychopathology Schizophrenia Research, 18, 21-28.

\section{_ , Hayward, P., Applethwaite, G., et al (1996)}

Compliance therapy in psychotic patients: randomised controlled trial. British Medical Journal, 312, 345-349.

Kim, Y., Sakamoto, K., Kamo, T., et al (1997) Insight and clinical correlates in schizophrenia. Comprehensive Psychiatry, 38, 117-123.

Liddle, P. F. (1987) Schizophrenic syndromes, cognitive performance and neurological dysfunctions.

Psychological Medicine I7, 49-57.

López-lbor, J. J. (1980) El sisterna AMDP. Madrid: Garsi.

Lysaker, P. H., Bell, M. D., Bryson, G., et al (1999) Personality as a predictor of the variability of insight in schizophrenia. Journal of Nervous and Mental Disease, 187. $119-122$.

McEvoy, J. P., Aland, J., Wilson, W. H., et al (198I) Measuring chronic schizophrenic patients' attitudes toward their illness and treatment. Hospital \& Community Psychiatry, 32, 856-858.

\section{CLINICAL IMPLICATIONS}

A 'semi-independent model' is proposed to explain longitudinal relationships between insight and psychopathological dimensions.

- Certain aspects of insight into past episodes improved over time in psychosis, regardless of diagnosis.

- Providing that different insight scales measure the same construct, the choice of a particular insight scale should depend on the aims and preferences of investigators or clinicians.

\section{LIMITATIONS}

The rater who evaluated the Scale to Assess Unawareness of Mental Disorder also took part in the evaluation of insight by means of the Manual for Assessment and Documentation in Psychopathology inventory.

- Results concerning relationships between insight and psychopathological dimensions should be considered as exploratory, since a liberal statistical criterion was chosen.

One-quarter of patients left the study, which limited the generalisability of our results to the whole population with psychosis.

MANUEL J. CUESTA, MD, VICTOR PERALTA, MD, AMALIA ZARZUELA, PhD, Psychiatric Unit, 'Virgen del Camino' Hospital, Pamplona, Spain

Correspondence: Dr Manuel J. Cuesta, Psychiatric Unit of 'Virgen del Camino' Hospital, E-31008 Pamplona, Spain. Fax +34948 429924; e-mail: mj.cuesta.zorita@cfnavarra.es

(First received 3 December 1999, final revision 23 February 2000, accepted 24 March 2000)

—, Apperson, L. J., Appelbaum, P. S., et al (1989a) Insight in schizophrenia. Its relationship to acute psychopathology. Journal of Nervous and Mental Disease, 177, 43-47.

_ , Freter, S., Everett, G., et al (1989b) Insight and clinical outcome of schizophrenic patients. Journal of Nervous and Mental Disease, I77, 48-5!.

Peralta, V., de Leon, J. \& Cuesta, M. J. (1992) Are there more than two syndromes in schizophrenia? A critique of the positive-negative dichotomy. British Journal of Psychiatry, 161, 335-343.

_ \& Cuesta, M. J. (1994) Lack of insight: its status within schizophrenic psychopathology. Biological Psychiatry, 36, 559-561.

Salvador-Carulla, L. (1996) Assessment instruments in psychiatry: description and psychometric properties. In Mental Health Outcome Measures (eds G. Thornicroft \& M. Tansella), pp. 179-206. Berlin: Springer.
Sanz, M., Constable, G., Lopez Ibor, I., et al (1998) A comparative study of insight scales and their relationship to psychopathological and clinical variables. Psychological Medicine, 28, 437-446.

Smith, T. E., Hull, J. W. \& Santos, L. (1998) The relationship between symptoms and insight in schizophrenia: a longitudinal perspective. Schizophrenia Research, 33, 63-67.

van Os, J., Fahy, T., Jones, P., et al (1996)

Psychopathological syndromes in the functional psychoses: associations with course and outcome. Psychological Medicine, 26, 161-176.

World Health Organization (1973) The Internationa Pilot Study of Schizophrenia, Vol. I. (WHO Offset Publication no. 2). Geneva: WHO. 